\title{
Beneficial Effects of the Free Radical Scavenger Edaravone (Radicut) in Neurologic Diseases
}

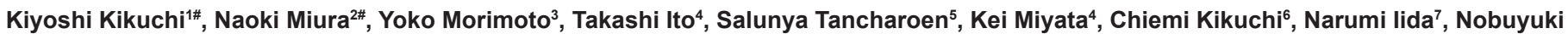
Takeshige $^{8}$, Hisaaki Uchikado ${ }^{8}$, Naohisa Miyagi ${ }^{11}$, Naoto Shiomi ${ }^{9}$, Terukazu Kuramoto ${ }^{10}$, Teruto Hashiguchi ${ }^{4}$, Ikuro Maruyama ${ }^{4}$, Motohiro Morioka ${ }^{8}$ and Ko-ichi Kawahara ${ }^{4,11 *}$

${ }^{1}$ Department of Neurosurgery, Yame Public General Hospital, 540-2 Takatsuka, Yame 834-0034, Japan

${ }^{2}$ Veterinary Teaching Hospital and Laboratory of Veterinary Diagnostic Imaging, Faculty of Agriculture, Kagoshima University, 1-21-24 Korimoto, Kagoshima 890-0065, Japan

${ }^{3}$ Department of Restorative Dentistry and Endodontology, Kagoshima University Graduate School of Medical and Dental Sciences, 8-35-1 Sakuragaoka, Kagoshima 8908544, Japan

${ }^{4}$ Division of Laboratory and Vascular Medicine, Field of Cardiovascular and Respiratory Disorders, Department of Advanced Therapeutics, Kagoshima University

Graduate School of Medical and Dental Sciences, 8-35-1 Sakuragaoka, Kagoshima 890-8520, Japan

${ }^{5}$ Department of Pharmacology, Faculty of Dentistry, Mahidol University, 6 Yothe Road, Rajthevee, Bangkok 10400, Thailand

${ }^{6}$ Department of Rehabilitation, Nishida Koutoku Hospital, 2-266 Tsuruokanishimachi, Saiki, Oita 876-0047, Japan

${ }^{7}$ Research Laboratory, Kohjin Co., Ltd., 1-6 Higashihama, Saiki, Oita 876-8580, Japan

${ }^{8}$ Department of Neurosurgery, Kurume University School of Medicine, 67 Asahimachi, Kurume 830-0011, Japan

${ }^{9}$ Department of Emergency, Saiseikai Shiga Hospital, 2-4-1 Ohashi, Rittou, Shiga 520-3046, Japan

${ }^{10}$ Department of Neurosurgery, Omuta City Hospital, 2-19-1 Takarazaka, Omuta, Fukuoka 836-8567, Japan

${ }^{11}$ Laboratory of Functional Foods, Department of Biomedical Engineering Osaka Institute of Technology, 5-16-1 Omiya, Asahi Ward, Osaka 535-8585, Japan

\#These Authors are Contributed equally

\begin{abstract}
Free radicals play major roles in the pathogenesis of many diseases, including neurologic diseases, making them an attractive target for therapeutic intervention. Several free radical scavengers have been developed, and some have progressed to clinical trials for the treatment of ischemic stroke. One such scavenger, edaravone is currently used to treat patients who present within $24 \mathrm{~h}$ of an attack. Edaravone can diffuse into many affected organs. Edaravone also exerts protective effects against brain and spinal cord injuries. Beyond its direct free radical scavenging effect, edaravone has anti-apoptotic and anti-inflammatory effects in various diseases. Here, we critically review the literature on experimental animal model and clinical studies of edaravone efficacy, and examine whether it should be considered a candidate for worldwide development. Edaravone has proven safe during 10 years of use as a free radical scavenger to treat ischemic stroke. In addition to ischemic stroke treatment, animal data suggest that edaravone may be an effective treatment option for several neurologic diseases, but additional clinical trials are necessary to verify its efficacy.
\end{abstract}

\section{Introduction}

The World Health Organization estimates that 15 million people suffer strokes worldwide annually. More than five million die from the initial brain insult, and another five million are permanently disabled and require lifelong medical care (www.strokecenter.org/patients/stats. htm). The National Institute of Neurological Disorders and Stroke (NINDS) classifies ischemic stroke types and determines incidence rates from population-based studies [1,2]. The NINDS estimates that cardioembolic stroke caused by an embolism from a cardiac source accounts for $29 \%$ of cases. Atheroembolic stroke, which is associated with narrowing of a cervicocephalic artery, makes up $16 \%$ of cases. Small vessel lacunar stroke, defined as pure motor, sensorimotor or sensory deficits with ataxic hemiparesis, results from thrombosis in one of the deep penetrating branches of the large cerebral arteries and accounts for $16 \%$ of cases [3]. Approximately $40 \%$ of ischemic strokes have an unknown cause [3]. Because oxidative stress is a major component of the ischemic stroke cascade [4], removal of free radicals may offer therapeutic benefits.

Several free radical scavengers have been assessed for their efficacy in the treatment of ischemic stroke, but few of these have shown success in studies conducted in Western countries [3]. In contrast, trials conducted in Japan have been more successful [3]. Indeed, several free radical scavengers have now been developed, and some of these (e.g., tirilazad and NXY-059) have progressed to clinical trials [5]. However, tirilazad produced inadequate therapeutic effects in patients with ischemic stroke and the trial was terminated. The Stroke-Acute
Ischemic NXY-059 Treatment (SAINT)-II trial found that NXY-059 was ineffective against ischemic stroke when administered within $4 \mathrm{~h}$ (mean time from onset of stroke to treatment, $3 \mathrm{~h} 46 \mathrm{~min}$ ) after the onset of symptoms [6-8].

The free radical scavenger edaravone (3-methyl-1-phenyl-2pyrazolin-5-one, MCI-186, Radicut; Mitsubishi Tanabe Pharma Corporation, Osaka, Japan) exerts antioxidant effects by inhibiting hydroxyl radical-dependent and -independent lipid peroxidation $[9,10]$. This antioxidant activity, the main proposed mechanism of action, may protect against free radical-related injuries following ischemic stroke [11]. Edaravone also suppresses the increase in hydroxyl and superoxide anion radical levels in several models of ischemic stroke $[12,13]$. Unlike other free radical scavengers, edaravone readily crosses

*Corresponding author: Ko-ichi Kawahara Phd., Division of Laboratory and Vascular Medicine, Department of Advanced Therapeutics, Kagoshima University Graduate School of Medical and Dental Sciences, 8-35-1 Sakuragaoka, Kagoshima 890-8520, Japan, Tel: +81-99-275-5437; Fax: +8199-275-2629; E-mail: telo@m3.kufm.kagoshima-u.ac.jp

Received May 11, 2011; Accepted June 30, 2011; Published September 20, 2011

Citation: Kikuchi K, Miura N, Morimoto Y, Ito T, Tancharoen S, et al. (2011) Beneficial Effects of the Free Radical Scavenger Edaravone (Radicut) in Neurologic Diseases. J Neurol Neurophysiol S1. doi:10.4172/2155-9562.S1-001

Copyright: ( 2011 Kikuchi K, et al. This is an open-access article distributed under the terms of the Creative Commons Attribution License, which permits unrestricted use, distribution, and reproduction in any medium, provided the original author and source are credited. 
the blood-brain barrier (BBB) [3], possibly explaining its efficacy where other scavengers have failed to show any.

In addition to its free radical scavenging effects, edaravone exerts unrelated effects that may be useful in the treatment of diseases other than ischemic stroke. To the best of our knowledge, no reports have reviewed edaravone's potential use in the treatment of other neurologic diseases.

Therefore, the aims of this review are to present current research on the use of edaravone, primarily in animal models of various neurologic diseases, and to introduce a clinical study aimed at assessing the potential use of edaravone in the treatment of these diseases.

\section{Edaravone efficacy in ischemic stroke: Experimental studies}

Edaravone exerts its antioxidant effects by quenching the hydroxyl radical and inhibiting hydroxyl radical-dependent and hydroxyl radical-independent lipid peroxidation in both a focal ischemic model and a global ischemic model $[9,10,14,15]$. In early studies of the antioxidant activity of edaravone, its pKa was found to be 7.0, and the rate of oxidation initiated with an azo compound was positively correlated with $\mathrm{pH}$ [15]. At $50 \mu \mathrm{M}$, edaravone inhibited the aerobic oxidation of unilamellar soybean phosphatidylcholine liposomal membranes initiated with either a water-soluble or a lipidsoluble initiator [15]. A more recent study described the ability of edaravone to inhibit copper- and human umbilical vein endothelial cell (HUVEC)-mediated low-density lipoprotein (LDL) oxidation [14]. In rats, the mechanism of action of edaravone was found to involve an enhancement of endothelial nitric oxide synthase (eNOS) expression in HUVECs through stabilizing eNOS mRNA and reversing the inhibitory effect of oxidized LDL on eNOS expression [14]. The authors of that study speculated that the upregulation of eNOS and the decrease in LDL oxidation caused by edaravone may improve vascular blood flow, which may in turn have a protective effect in ischemic tissues. Additional studies have shown that edaravone suppresses the increases in hydroxyl radical and superoxide anion radical levels in both a focal ischemic model and a global ischemic model $[10,16,17]$.

Many effects of edaravone have been reported in basic studies of ischemic stroke. Reactive oxygen species (ROS) and $\mathrm{Ca}^{2+}$ overload during ischemia/reperfusion induce cellular damage by opening the mitochondrial permeability transition pore, a non-specific pore in the inner mitochondrial membrane [18]. Notably, edaravone attenuated $\mathrm{Ca}^{2+}$ induced swelling of mitochondria in the rat brain [18]. In a mouse focal ischemic model, the neuroprotective effects of edaravone were mediated via its antioxidant actions, including suppression of lipid peroxidation and oxidant DNA damage [19]. Edaravone also suppressed inducible nitric oxide synthase (iNOS) activity, thereby exerting anti-inflammatory effects by inhibiting microglial activity and peroxynitrite production [19]. In addition, data show that edaravone protects ischemic neurons from apoptosis by suppressing the expression of Fas-associated death domain protein, death-associated protein and caspase- 8 immunoreactivity in a rat middle cerebral artery occlusion (MCAO) model, a focal ischemic model [20]. Edaravone has also been shown to have an anti-apoptotic effect mediated by a decrease in the level of B-cell lymphoma 2 (Bcl-2)-associated X protein immunoreactivity and an increase in the levels of the apoptosis regulator Bcl-2 immunoreactivity in a rat MCAO model [21]. The protective effect of edaravone in hypoxic/ischemic injury has also been attributed to inhibition of response to endoplasmic reticulum (ER) stress and subsequent apoptotic signaling in a focal ischemic model
$[22,23]$. Using a standard ischemia model in gerbils, Jin and colleagues showed that edaravone could reduce edema and increase cerebral blood flow following ischemia [24]. They also showed that that edaravone and argatroban (a selective thrombin inhibitor and an anticoagulant agent used to treat acute noncardioembolic ischemic stroke) protect against damage to neuronal cells and increase the survival ratio $(p<0.05$ by Mantel-Cox test) [24], while another study revealed that edaravone can ameliorate damage when used in conjunction with argatroban [25]. Data from several animal models suggest that edaravone can suppress brain edema in hypoxic/ischemic conditions [26,27]. This effect is attributed to edaravone-mediated inhibition of vascular endothelial growth factor (VEGF) expression in astrocytes [26]. In addition to the inhibition of ROS generation, edaravone reduces the amount of ROSinduced inflammatory reactions in ischemic stroke [28]. Oxidative stress activates nuclear factor $-\kappa \mathrm{B}(\mathrm{NF}-\kappa \mathrm{B})$ and several mediators of inflammation (e.g., iNOS, cytokines and cyclocygenase-2) that are known to cause delayed damage to the ischemic area in stroke patients and models of stroke [29-36]. In cases of ischemic injury, edaravone can also reduce iNOS expression and suppress neutrophil activation and the accumulation of lipid peroxidation products and 4-hydroxy-2nonenal (HNE)-modified proteins [19,37].

Recent discoveries indicate many new benefits of edaravone in ischemic stroke. Several lines of evidence show neuroprotection after ischemic stroke. For example, ischemic stroke is associated with enhanced expression of metalloproteinase-9 (MMP-9) and aquaporin-4 (AQP4), which cause acute edema. It is also associated with the release of high-mobility group box 1 (HMGB1) from affected tissue. These events are associated with poor clinical outcomes $[3,24,38$ 40]. Edaravone is a low molecular weight agent that readily crosses the $\mathrm{BBB}$, and its activity is not limited to the vascular compartment $[41,42]$. Furthermore, edaravone was reported to inhibit MMP-9-related brain hemorrhage in rats treated with recombinant tissue plasminogen activator (tPA) [40] and to attenuate cerebral ischemic injury by suppressing AQP4 expression in a focal ischemia model [27]. Moreover, edaravone rescues rats from ischemic stroke by attenuating the release of HMGB1 from neuronal cells in a focal ischemia model [38]. Taken together, these findings suggest that edaravone could be used to treat ischemic stroke by targeting and inhibiting the underlying molecular events associated with brain injury. In a tPA-treated rat MCAO model, edaravone prevented the dissociation of the neurovascular unit (e.g. neurons, glia and vascular cells), dramatically decreased hemorrhagic transformation (hemorrhages that develop inside areas of ischemia), and improved neurologic scores and survival rate[43].

\section{Edaravone efficacy in ischemic stroke: Clinical studies}

Edaravone was the first free radical scavenger developed as a neuroprotective drug to be introduced worldwide. Since 2001, it has been used in Japan to treat many patients with ischemic stroke $[44,45]$. It is currently approved only in Japan. Clinical trial data show that administration of edaravone within $72 \mathrm{~h}$ of ischemic stroke onset significantly reduces infarct volume and provides sustained benefits over a 3-month follow-up period [46]. Administration of edaravone within $24 \mathrm{~h}$ of ischemic stroke onset has also been performed in patients with lacunae, large-artery atherosclerosis, and cardioembolic stroke as shown by the beneficial effects on rehabilitation $[47,48]$. In a retrospective study of 72 acute ischemic stroke patients, Unno et al. reported that the total dose of edaravone was associated with gains in rehabilitation [47]. Furthermore, in a randomized, controlled pilot study of 41 acute ischemic stroke patients, edaravone treatment for 
up to 14 days slowed the progression of disuse muscle atrophy and improved leg locomotor function to a greater extent than shorterterm treatment (3 day treatment) [48]. Moreover, Shinohara and coworkers studied the effects of edaravone in a mixed population of 401 acute ischemic stroke patients including patients with thrombotic and lacunar stroke [25]. The study was part of a multicenter, randomized, parallel-group, open-label design comparing edaravone with sodium ozagrel (ozagrel) [25], an antiplatelet agent restricted to use in the treatment of acute noncardioembolic ischemic stroke[25]. The main conclusion was that edaravone was at least as effective as ozagrel [25]. These data showed that there was a trend toward decreased National Institutes of Health Stroke Scale scores in edaravone-treated patients. Furthermore, edaravonesuppressed reduced serum MMP9 levels in 63 patients with acute ischemic stroke in another randomized study [49].

\section{Edaravone efficacy in amyotrophic lateral sclerosis: Experimental studies}

Amyotrophic lateral sclerosis (ALS) is a devastating neurodegenerative disease involving selective and progressive degeneration and disappearance of motor neurons. Since $\mathrm{Cu} / \mathrm{Zn}$ dependent superoxide dismutase (SOD-1) was first identified as a contributing factor in familial ALS (FALS) in 1993, the possibility of a role of oxidative stress in the pathogenesis of FALS has been studied [50]. Oxidative lesions have been found in the nervous tissue of both sporadic ALS and FALS patients [51], and oxidative stress has been shown to contribute to the pathogenesis of ALS [52,53]. Mutant SOD1 transgenic mice also recapitulate the clinical symptoms and pathological findings of human FALS [54]. Beneficial effects of edaravone in mutant SOD1 mice in randomized experiments with a blinded design have been reported [55]. The deposition of abnormal SOD1 in the anterior horns was reduced by edaravone administration, and edaravone effectively slowed symptom progression and motor neuron degeneration [55]. Whereas FALS is well represented by transgenic mutant SOD1 mouse models, the mouse mutant 'wobbler' develops progressive motor neuron degeneration as a result of a point mutation in the Vps54 gene, and provides an animal model for sporadic ALS [56]. There is evidence of increased oxidative stress in the spinal cords of wobbler mice, and beneficial effects of edaravone in these mice have been reported [57].

\section{Edaravone efficacy in amyotrophic lateral sclerosis: Clinical studies}

Yoshino and Kimura reported the results of a small-sized open trial of edaravone in 19 ALS patients [58]. Edaravone markedly reduced the concentration of 3-nitrotyrosine, which is indicative of oxidative cellular damage and is increased in sporadic ALS patients [52], in the cerebrospinal fluid of enrolled patients. In addition, edaravone reduced the rate of decline in ALS functional rating scale scores during the sixmonth treatment period. Thus, treatment with edaravone may delay the progression of functional motor disturbances in ALS patients [58]. More promising evidence of the beneficial effects of edaravone in human ALS patients is expected with the publication of the results of a phase III clinical trial of edaravone in ALS patients, currently underway in Japan [59].

\section{Edaravone efficacy in traumatic brain injury: Experimental and clinical studies}

Like acute ischemic stroke, a large number of studies have reported that oxidative stress has a key role in the development of traumatic brain injury (TBI). Edaravone administration inhibited free radicalinduced neuronal degeneration and apoptotic cell death around the injured area, and improved cerebral dysfunction in a rat TBI model in which injury was introduced with a pneumatic controlled injury device [60]. Moreover, edaravone scavenged alkoxyl radicals in a rat cryoinjury TBI model [61], and, in a different study, increased neural stem cell numbers around the area of damage following TBI in rats [62]. Edaravone was also shown to scavenge alkoxyl radicals in 17 human patients with TBI [63]. However, neither neurologic function nor outcome was evaluated in this study.

\section{Edaravone efficacy in other neurologic diseases: Experimental studies}

A neuroprotective effect of edaravone was observed in a Parkinson's disease (PD) rat model, both in vitro and in vivo [64]. The rat PD model was produced by administration of 6-hydroxydopamine, a neurotoxin targeting dopaminergic neurons. The authors reported reductions in the numbers of terminal deoxynucleotidyl transferasemediated biotinylated UTP nick end labeling-positive apoptotic cells and hydroethidine-positive cells, suggesting that edaravone may have anti-apoptotic and anti-oxidative effects [64]. Staining for ionized calcium binding adaptor molecule 1 in a PD rat model revealed that inflammation was also suppressed after edaravone administration [64]. Edaravone also protects neurons from apoptosis after cranial irradiation and protects against spatial memory retention deficits in mice [65]. In addition, edaravone has been shown to protect human neural stem cells from radiation-induced apoptosis [30].

The neuroprotective effects of edaravone extend to spinal cord damage models. Edaravone administration reduced eNOS and SOD1 levels after transient ischemia in rabbits (a rabbit MCAO model) [66]. Furthermore, edaravone reduced oxidative DNA damage, as shown by the prolonged expression of the redox effector factor Raf1, a multifunctional enzyme involved in the DNA repair process, in the spinal cord of the same rabbit transient ischemia model [67]. Moreover, evaluation of the effect of edaravone on lipid peroxide formation downstream of the ROS production cascade, by measuring malonyldialdehyde levels in injured spinal cord homogenates, found that edaravone significantly attenuated lipid peroxide formation by $>45 \%$ in the acute stage of spinal cord injury in rats [68].

Anti-inflammatory effects of edaravone have been reported in a few experimental studies. In a murine experimental multiple sclerosis model, edaravone significantly ameliorated the clinical severity, reduced infiltration of lymphocytes and lowered iNOS expression [69]. Edaravone effectively inhibited NF-kB activation and decreased expression of chemoattractant protein-1, vascular cell adhesion molecular-1 and matrix metalloproteinase-2, resulting in significant inhibition of macrophage infiltration into aneurysmal walls of a rat cerebral aneurysm (CA) model generated by artery ligation [70]. Edaravone also inhibited enlargement and medial degradation of CAs without influencing systemic blood pressure in this rat model [70] These results suggest the possibility of a preventive effect of edaravone against CA rupture.

Finally, edaravone was shown to attenuate intracerebral hemorrhage-induced brain edema, neurologic deficits and oxidative injury in rats [71]. Furthermore, edaravone reduced iron- and thrombin-induced brain injury in rats [71]. A clear and selective inhibitory effect of edaravone against hydroxyl radical-induced vasoconstriction was shown in the canine basilar artery in vitro [72].

\section{Conclusions}

The findings of recent research on the efficacy and mechanism of action of edaravone suggest tremendous potential for this scavenger 
Citation: Kikuchi K, Miura N, Morimoto Y, Ito T, Tancharoen S, et al. (2011) Beneficial Effects of the Free Radical Scavenger Edaravone (Radicut) in Neurologic Diseases. J Neurol Neurophysiol S1. doi:10.4172/2155-9562.S1-001

in the treatment of several neurologic diseases. However, edaravone is currently only used to treat ischemic stroke within Japan. The abnormal generation of free radicals appears to be common to the etiology and progression of multiple diseases, in particular, a variety of neurologic diseases. Therefore, edaravone may have potential therapeutic effects in patients with neurologic diseases. In addition, edaravone has been shown to have many effects beyond free radical scavenging, such as anti-apoptotic and anti-cytokine effects, in animal models of various neurologic diseases. To date, the therapeutic effects of edaravone in humans have only been reported in ALS and ischemic stroke patients. Further clinical studies are desired to extend the effects of edaravone seen in various animal models of disease to humans.

\section{References}

1. Petty GW, Brown RD, Whisnant JP, Sicks JD, O'Fallon WM, et al. (1999) Ischemic stroke subtypes: a population-based study of incidence and risk factors. Stroke 30: 2513-2516.

2. Petty GW, Brown RD, Whisnant JP, Sicks JD, O'Fallon WM, et al. (2000) Ischemic stroke subtypes: a population-based study of functional outcome, survival, and recurrence. Stroke 31: 1062-1068.

3. Lapchak PA, Zivin JA (2009) The lipophilic multifunctional antioxidant edaravone (radicut) improves behavior following embolic strokes in rabbits: a combination therapy study with tissue plasminogen activator. Exp Neurol 215: 95-100.

4. Lapchak PA, Araujo DM (2007) Advances in ischemic stroke treatment: neuroprotective and combination therapies. Expert Opin Emerg Drugs 12: $97-$ 112.

5. Wang CX, Shuaib A (2007) Neuroprotective effects of free radical scavengers in stroke. Drugs Aging 24: 537-546.

6. Green AR, Shuaib A (2006) Therapeutic strategies for the treatment of stroke. Drug Discov Today 11: 681-693.

7. Van Der Worp HB, Kappelle LJ, Algra A, Bar PR, Orgogozo JM, et al. (2002) The effect of tirilazad mesylate on infarct volume of patients with acute ischemic stroke. Neurology 58: 133-135.

8. Shuaib A, Lees KR, Lyden P, Grotta J, Davalos A, et al. (2007) NXY-059 for the treatment of acute ischemic stroke. N Engl J Med 357: 562-571.

9. Watanabe T, Yuki S, Egawa M, Nishi H (1994) Protective effects of $\mathrm{MCl}-186$ on cerebral ischemia: possible involvement of free radical scavenging and antioxidant actions. J Pharmacol Exp Ther 268: 1597-1604.

10. Yamamoto T, Yuki S, Watanabe T, Mitsuka M, Saito KI, et al. (1997) Delayed neuronal death prevented by inhibition of increased hydroxyl radical formation in a transient cerebral ischemia. Brain Res 762: 240-242.

11. Lapchak PA, Araujo DM (2003) Development of the nitrone-based spin trap agent NXY-059 to treat acute ischemic stroke. CNS Drug Rev 9: 253-262.

12. Hoehn B, Yenari MA, Sapolsky RM, Steinberg GK (2003) Glutathione peroxidase overexpression inhibits cytochrome $C$ release and proapoptotic mediators to protect neurons from experimental stroke. Stroke 34: 2489-2494.

13. Okatani Y, Wakatsuki A, Kaneda C (2000) Melatonin increases activities of glutathione peroxidase and superoxide dismutase in fetal rat brain. $\mathrm{J}$ Pineal Res 28: 89-96

14. Yoshida H, Sasaki K, Namiki Y, Sato N, Tada N (2005) Edaravone, a novel radical scavenger, inhibits oxidative modification of low-density lipoprotein (LDL) and reverses oxidized LDL-mediated reduction in the expression of endothelial nitric oxide synthase. Atherosclerosis 179: 97-102.

15. Yamamoto Y, Kuwahara T, Watanabe K, Watanabe K (1996) Antioxidant activity of 3-methyl-1-phenyl-2-pyrazolin-5-one. Redox Report 2: 333-338.

16. Mizuno A, Umemura K, Nakashima M (1998) Inhibitory effect of $\mathrm{MCl}-186$, a free radical scavenger, on cerebral ischemia following rat middle cerebral artery occlusion. Gen Pharmacol 30: 575-578.
17. Shichinohe H, Kuroda S, Yasuda H, Ishikawa T, Iwai M, et al. (2004) Neuroprotective effects of the free radical scavenger Edaravone (MCl-186) in mice permanent focal brain ischemia. Brain Res 1029: 200-206.

18. Takayasu Y, Nakaki J, Kawasaki T, Koda K, Ago Y, et al. (2007) Edaravone, a radical scavenger, inhibits mitochondrial permeability transition pore in rat brain. J Pharmacol Sci 103: 434-437.

19. Zhang N, Komine-Kobayashi M, Tanaka R, Liu M, Mizuno Y, et al. (2005) Edaravone reduces early accumulation of oxidative products and sequentia inflammatory responses after transient focal ischemia in mice brain. Stroke 36 : 2220-2225.

20. Xiao B, Bi FF, Hu YQ, Tian FF, Wu ZG, et al. (2007) Edaravone neuroprotection effected by suppressing the gene expression of the Fas signal pathway following transient focal ischemia in rats. Neurotox Res 12: 155-162.

21. Amemiya S, Kamiya T, Nito C, Inaba T, Kato K, et al. (2005) Anti-apoptotic and neuroprotective effects of edaravone following transient focal ischemia in rats. Eur J Pharmacol 516: 125-130.

22. Qi X, Okuma Y, Hosoi T, Nomura Y (2004) Edaravone protects against hypoxia/ ischemia-induced endoplasmic reticulum dysfunction. J Pharmacol Exp Ther 311: 388-393.

23. Yung HW, Korolchuk S, Tolkovsky AM, Charnock-Jones DS, Burton GJ (2007) Endoplasmic reticulum stress exacerbates ischemia-reperfusioninduced apoptosis through attenuation of Akt protein synthesis in human choriocarcinoma cells. FASEB J 21: 872-884.

24. Jin YJ, Mima T, Raicu V, Park KC, Shimizu K (2002) Combined argatroban and edaravone caused additive neuroprotection against $15 \mathrm{~min}$ of forebrain ischemia in gerbils. Neurosci Res 43: 75-79.

25. Shinohara Y, Saito I, Kobayashi S, Uchiyama S (2009) Edaravone (radical scavenger) versus sodium ozagrel (antiplatelet agent) in acute noncardioembolic ischemic stroke (EDO trial). Cerebrovasc Dis 27: 485-492.

26. Ishikawa A, Yoshida H, Metoki N, Toki T, Imaizumi T, et al. (2007) Edaravone inhibits the expression of vascular endothelial growth factor in human astrocytes exposed to hypoxia. Neurosci Res 59: 406-412.

27. Kikuchi K, Tancharoen S, Matsuda F, Biswas KK, Ito T, et al. (2009) Edaravone attenuates cerebral ischemic injury by suppressing aquaporin-4. Biochem Biophys Res Commun 390: 1121-1125.

28. Barone FC, Feuerstein GZ (1999) Inflammatory mediators and stroke: new opportunities for novel therapeutics. J Cereb Blood Flow Metab 19: 819-834.

29. Baird AE, Benfield A, Schlaug G, Siewert B, Lovblad KO, et al. (1997) Enlargement of human cerebral ischemic lesion volumes measured by diffusion-weighted magnetic resonance imaging. Ann Neurol 41: 581-589.

30. Bemeur C, Ste-Marie L, Desjardins P, Vachon L, Butterworth RF, et al (2005) Dehydroascorbic acid normalizes several markers of oxidative stress and inflammation in acute hyperglycemic focal cerebral ischemia in the rat Neurochem Int 46: 399-407.

31. Block F, Dihne M, Loos M (2005) Inflammation in areas of remote changes following focal brain lesion. Prog Neurobiol 75: 342-365.

32. Bramlett HM, Dietrich WD (2004) Pathophysiology of cerebral ischemia and brain trauma: similarities and differences. J Cereb Blood Flow Metab 24: 133150.

33. Khan M, Sekhon B, Jatana M, Giri S, Gilg AG, et al. (2004) Administration of $\mathrm{N}$-acetylcysteine after focal cerebral ischemia protects brain and reduces inflammation in a rat model of experimental stroke. J Neurosci Res 76: 519527

34. Kim JB, Yu YM, Kim SW, Lee JK (2005) Anti-inflammatory mechanism is involved in ethyl pyruvate-mediated efficacious neuroprotection in the postischemic brain. Brain Res 1060: 188-192.

35. Matsui T, Mori T, Tateishi N, Kagamiishi Y, Satoh S, et al. (2002) Astrocytic activation and delayed infarct expansion after permanent focal ischemia in rats. Part I: enhanced astrocytic synthesis of s-100beta in the periinfarct area precedes delayed infarct expansion. J Cereb Blood Flow Metab 22: 711-722. 
Citation: Kikuchi K, Miura N, Morimoto Y, Ito T, Tancharoen S, et al. (2011) Beneficial Effects of the Free Radical Scavenger Edaravone (Radicut) in Neurologic Diseases. J Neurol Neurophysiol S1. doi:10.4172/2155-9562.S1-001

36. Wen Y, Yang S, Liu R, Perez E, Yi KD, et al. (2004) Estrogen attenuates nuclear factor-kappa $\mathrm{B}$ activation induced by transient cerebral ischemia. Brain Res 1008: 147-154.

37. Suzuki YJ, Forman HJ, Sevanian A (1997) Oxidants as stimulators of signal transduction. Free Radic Biol Med 22: 269-285.

38. Kikuchi K, Kawahara K, Tancharoen S, Matsuda F, Morimoto Y, et al. (2009) The free radical scavenger edaravone rescues rats from cerebral infarction by attenuating the release of high-mobility group box- 1 in neuronal cells. J Pharmacol Exp Ther 329: 865-874.

39. Papadopoulos MC, Krishna S, Verkman AS (2002) Aquaporin water channels and brain edema. Mt Sinai J Med 69: 242-248.

40. Yagi K, Kitazato KT, Uno M, Tada Y, Kinouchi T, et al. (2009) Edaravone, a free radical scavenger, inhibits MMP-9-related brain hemorrhage in rats treated with tissue plasminogen activator. Stroke 40: 626-631.

41. Higashi Y, Jitsuiki D, Chayama K, Yoshizumi M (2006) Edaravone (3-methyl1-phenyl-2-pyrazolin-5-one), a novel free radical scavenger, for treatment of cardiovascular diseases. Recent Pat Cardiovasc Drug Discov 1: 85-93.

42. Yoshida H, Yanai H, Namiki Y, Fukatsu-Sasaki K, Furutani N, et al. (2006) Neuroprotective effects of edaravone: a novel free radical scavenger in cerebrovascular injury. CNS Drug Rev 12: 9-20.

43. Yamashita T, Kamiya T, Deguchi K, Inaba T, Zhang H, et al. (2009) Dissociation and protection of the neurovascular unit after thrombolysis and reperfusion in ischemic rat brain. J Cereb Blood Flow Metab 29: 715-725.

44. Adams HP, Jr., Del Zoppo G, Alberts MJ, Bhatt DL, Brass L, et al. (2007) Guidelines for the early management of adults with ischemic stroke: a guideline from the American Heart Association/American Stroke Association Stroke Council, Clinical Cardiology Council, Cardiovascular Radiology and Intervention Council, and the Atherosclerotic Peripheral Vascular Disease and Quality of Care Outcomes in Research Interdisciplinary Working Groups: the American Academy of Neurology affirms the value of this guideline as an educational tool for neurologists. Stroke 38: 1655-1711.

45. Yoneda Y, Uehara T, Yamasaki H, Kita Y, Tabuchi M, et al. (2003) Hospitalbased study of the care and cost of acute ischemic stroke in Japan. Stroke 34 : 718-724.

46. Edaravone Acute Infarction Study Group (2003) Effect of a novel free radical scavenger, edaravone (MCl-186), on acute brain infarction. Randomized, placebo-controlled, double-blind study at multicenters. Cerebrovasc Dis 15: 222-229.

47. Unno Y, Katayama M, Shimizu H (2010) Does functional outcome in acute ischaemic stroke patients correlate with the amount of free-radical scavenger treatment? A retrospective study of edaravone therapy. Clin Drug Investig 30: $143-155$

48. Naritomi H, Moriwaki H, Metoki N, Nishimura H, Higashi Y, et al. (2010) Effects of Edaravone on Muscle Atrophy and Locomotor Function in Patients with Ischemic Stroke: A Randomized Controlled Pilot Study. Drugs R D 10: 155-163.

49. Isahaya K, Yamada K, Yamatoku M, Sakurai K, Takaishi S, et al. (2011) Effects of Edaravone, a Free Radical Scavenger, on Serum Levels of Inflammatory Biomarkers in Acute Brain Infarction. J Stroke Cerebrovasc Dis. 2011 Jan 5.[Epub ahead of print]

50. Rosen DR, Siddique T, Patterson D, Figlewicz DA, Sapp P, et al. (1993) Mutations in $\mathrm{Cu} / \mathrm{Zn}$ superoxide dismutase gene are associated with familial amyotrophic lateral sclerosis. Nature 362: 59-62.

51. Ferrante RJ, Browne SE, Shinobu LA, Bowling AC, Baik MJ, et al. (1997) Evidence of increased oxidative damage in both sporadic and familial amyotrophic lateral sclerosis. Journal of neurochemistry 69: 2064-2074.

52. Beal MF, Ferrante RJ, Browne SE, Matthews RT, Kowall NW, et al. (1997) Increased 3-nitrotyrosine in both sporadic and familial amyotrophic lateral sclerosis. Annals of neurology 42: 644-654.

53. Shibata N (2001) Transgenic mouse model for familial amyotrophic latera sclerosis with superoxide dismutase- 1 mutation. Neuropathology: official journal of the Japanese Society of Neuropathology 21: 82-92.
54. Gurney ME, Pu H, Chiu AY, Dal Canto MC, Polchow CY, et al. (1994) Motor neuron degeneration in mice that express a human $\mathrm{Cu}, \mathrm{Zn}$ superoxide dismutase mutation. Science 264: 1772-1775.

55. Ito H, Wate R, Zhang J, Ohnishi S, Kaneko S, et al. (2008) Treatment with edaravone, initiated at symptom onset, slows motor decline and decreases SOD1 deposition in ALS mice. Exp Neurol 213: 448-455.

56. Palmisano R, Golfi P, Heimann P, Shaw C, Troakes C, et al. (2011) Endosomal accumulation of APP in wobbler motor neurons reflects impaired vesicle trafficking: implications for human motor neuron disease. BMC Neuroscience 12: 24 .

57. Ikeda K, Iwasaki Y, Kinoshita M (2010) Treatment of wobbler mice with free radical scavenger. Molecular mechanism and therapeutics of amyctrophic lateral sclerosis. 2001: 335-340.

58. Yoshino H, Kimura A (2006) Investigation of the therapeutic effects of edaravone, a free radical scavenger, on amyotrophic lateral sclerosis (Phase II study). Amyotroph Lateral Scler 7: 241-245.

59. Takahashi R (2009) Edaravone in ALS. Exp Neurol 217: 235-236.

60. Itoh T, Satou T, Nishida S, Tsubaki M, Imano M, et al. (2010) Edaravone protects against apoptotic neuronal cell death and improves cerebral function after traumatic brain injury in rats. Neurochem Res 35: 348-355.

61. Dohi K, Satoh K, Nakamachi T, Yofu S, Hiratsuka K, et al. (2007) Does edaravone $(\mathrm{MCl}-186)$ acts as an antioxidant and a neuroprotector in experimental traumatic brain injury? Antioxid Redox Signal 9: 281-287.

62. Itoh T, Satou T, Nishida S, Tsubaki M, Hashimoto S, et al. (2009) The nove free radical scavenger, edaravone, increases neural stem cell number around the area of damage following rat traumatic brain injury. Neurotox Res 16: 378389.

63. Dohi K, Satoh K, Mihara Y, Nakamura S, Miyake Y, et al. (2006) Alkoxyl radical-scavenging activity of edaravone in patients with traumatic brain injury. J Neurotrauma 23: 1591-1599.

64. Yuan WJ, Yasuhara T, Shingo T, Muraoka K, Agari T, et al. (2008) Neuroprotective effects of edaravone-administration on 6-OHDA-treated dopaminergic neurons. BMC Neurosci 9: 75.

65. Motomura K, Ogura M, Natsume A, Yokoyama H, Wakabayashi T (2010) A free-radical scavenger protects the neural progenitor cells in the dentate subgranular zone of the hippocampus from cell death after X-irradiation. Neurosci Lett 485: 65-70.

66. Takahashi G, Sakurai M, Abe K, Itoyama Y, Tabayashi K (2003) MCl-186 prevents spinal cord damage and affects enzyme levels of nitric oxide synthase and $\mathrm{Cu} / \mathrm{Zn}$ superoxide dismutase after transient ischemia in rabbits. $\mathrm{J}$ Thorac Cardiovasc Surg 126: 1461-1466.

67. Takahashi G, Sakurai M, Abe K, Itoyama Y, Tabayashi K (2004) MCl-186 reduces oxidative cellular damage and increases DNA repair function in the rabbit spinal cord after transient ischemia. Ann Thorac Surg 78: 602-607.

68. Ohta S, Iwashita Y, Takada H, Kuno S, Nakamura T (2005) Neuroprotection and enhanced recovery with edaravone after acute spinal cord injury in rats. Spine (Phila Pa 1976) 30: 1154-1158.

69. Moriya M, Nakatsuji Y, Miyamoto K, Okuno T, Kinoshita M, et al. (2008) Edaravone, a free radical scavenger, ameliorates experimental autoimmune encephalomyelitis. Neurosci Lett 440: 323-326.

70. Aoki T, Nishimura M, Kataoka H, Ishibashi R, Nozaki K, et al. (2009) Reactive oxygen species modulate growth of cerebral aneurysms: a study using the free radical scavenger edaravone and p47phox(-/-) mice. Lab Invest 89: 730-741.

71. Nakamura T, Kuroda Y, Yamashita S, Zhang X, Miyamoto O, et al. (2008) Edaravone attenuates brain edema and neurologic deficits in a rat model of acute intracerebral hemorrhage. Stroke 39: 463-469.

72. Tosaka M, Hashiba Y, Saito N, Imai H, Shimizu T, et al. (2002) Contractile responses to reactive oxygen species in the canine basilar artery in vitro: selective inhibitory effect of $\mathrm{MCl}-186$, a new hydroxyl radical scavenger. Acta Neurochir (Wien) 144: 1305-1310. 